\title{
BMJ Open Study protocol for the MEXiletine hydrochloride administration trial: a placebo-controlled, randomised, double- blind, multicentre, crossover study of its efficacy and safety in spinal and bulbar muscular atrophy (MEXPRESS)
}

Shinichiro Yamada, ${ }^{1}$ Atsushi Hashizume, ${ }^{1}$ Yasuhiro Hijikata, ${ }^{1}$ Tomonori Inagaki, ${ }^{1}$ Daisuke Ito, ${ }^{1}$ Fumie Kinoshita, ${ }^{2}$ Masahiro Nakatochi, ${ }^{2}$ Yumiko Kobayashi, ${ }^{2}$ Akihiro Hirakawa, ${ }^{3}$ Tomohiko Nakamura, ${ }^{1}$ Masahisa Katsuno ${ }^{1}$

To cite: Yamada S,

Hashizume A, Hijikata Y, et al. Study protocol for the MEXiletine hydrochloride administration trial: a placebo-controlled, randomised, double-blind, multicentre, crossover study of its efficacy and safety in spinal and bulbar muscular atrophy (MEXPRESS). BMJ Open 2018;8:e023041. doi:10.1136/ bmjopen-2018-023041

- Prepublication history for this paper is available online To view these files, please visit the journal online (http://dx.doi. org/10.1136/bmjopen-2018023041).

Received 19 March 2018 Revised 8 August 2018 Accepted 9 August 2018

Check for updates

(C) Author(s) (or their employer(s)) 2018. Re-use permitted under CC BY-NC. No commercial re-use. See rights and permissions. Published by BMJ.

For numbered affiliations see end of article.

Correspondence to Professor Masahisa Katsuno; ka2no@med.nagoya-u.ac.jp

\section{ABSTRACT}

Introduction Spinal and bulbar muscular atrophy (SBMA)

is a slowly progressive neuromuscular disease. Cold exposure often leads to worsening of motor symptoms including paresis. Although mexiletine hydrochloride administration has been shown to be effective for the treatment of several muscular diseases, its effectiveness in SBMA has not been validated to date. The trial will test it as a symptomatic drug for cold paresis. This study is the first trial to evaluate the efficacy and safety of mexiletine hydrochloride administration in patients with SBMA. Methods and analysis A placebo-controlled, randomised, double-blind, multicentre, crossover clinical trial will be conducted to assess the safety and efficacy of mexiletine hydrochloride in patients with SBMA. The eligible patients will be assigned randomly in a 1:1 ratio to two groups in a double-blind manner. Participants will take mexiletine hydrochloride ( $300 \mathrm{mg} /$ day) or a placebo orally three times a day for 4 weeks (period 1). After a 1-week washout period, participants will take the other drug for 4 weeks (period 2). The primary endpoint is the difference in distal latencies between room temperature and cold exposure conditions.

Ethics and dissemination This study will be conducted in compliance with the Helsinki Declaration and the Ethical Guidelines for Medical and Health Research Involving Human Subjects by the Japanese government and has been approved by the ethics committee of Nagoya University Graduate School of Medicine, as a central institutional review board, and by each facility. The results will be disseminated in peer-reviewed journals and at scientific conferences.

Trial registration number UMIN000026150; Pre-results.

\section{INTRODUCTION}

Spinal and bulbar muscular atrophy (SBMA), or Kennedy disease, is a slowly progressive lower motor neuron and muscular disease characterised by bulbar and limb muscle
Strengths and limitations of this study

- Cold paresis is a well-known symptom in patients with spinal and bulbar muscular atrophy (SBMA); however, no therapies that provide symptomatic relief are currently available.

- This trial is the first randomised controlled trial to evaluate the efficacy and safety of mexiletine hydrochloride in patients with SBMA.

- An objective index, a change in the difference in the distal latencies between room temperature and cold exposure conditions, has been adopted as the primary endpoint.

- It might be difficult to assess the questionnaire for paresis in summer, as cold paresis is likely to be mild with high temperature. Therefore, trial enrolment and duration may have to be regulated to ensure robustness of data.

weakness and elevated serum creatine kinase levels. $^{1-3}$ SBMA is caused by the expansion of a cytosine, adenine and guanine (CAG) repeat within the first exon of the androgen receptor $(A R)$ gene. ${ }^{4}$ The mutant $\mathrm{AR}$ protein induces degeneration of motor neurons and skeletal muscles. ${ }^{25}$ Muscular weakness generally appears between 30 and 60 years of age, and the affected subjects typically require a wheelchair 15 to 20 years after the onset of muscular weakness. ${ }^{2}$ Patients often experience muscular weakness or myotonia-like muscle contraction during cold exposure, which may result in a deterioration of activities of daily living (ADL). ${ }^{6}$

In patients with non-dystrophic myotonia caused by gene mutations that encode chloride and sodium channels on skeletal muscles, 
cold exposure is the most common trigger of muscular weakness and myotonia. ${ }^{7}$ Muscular weakness caused by cold exposure was also reported in patients with multifocal motor neuropathy. ${ }^{8}$ Although myotonia is rare in SBMA, muscular weakness during cold exposure often occur in patients with this disease. Given that cold paresis has been seen in myotonic disorders such as paramyotonia congenita, ${ }^{9}{ }^{10}$ SBMA and myotonic disorders may share common pathophysiology. Low temperatures slow both opening and closing of the $\mathrm{Na} / \mathrm{K}$ pump due to the thermal reduction of its ATPase activity, leading to a slow conduction velocity and weakness. ${ }^{1-13}$ Similarly, the depolarisation of muscle fibres depends on the influx of $\mathrm{Na}^{+}$ions; therefore, lower temperatures prolong the depolarisation of each muscle fibre potential. Cold paralysis and myotonia is caused by muscle membrane hyperexcitability ${ }^{13-15}$; several possible mechanisms may be involved, including a reduction in the resting chloride conductance and/or a gain of function in voltage-dependent sodium channels that lead to excessive sodium current in skeletal muscle. ${ }^{15}$

Therefore, due to the membrane hyperexcitability in SBMA, we hypothesised that voltage-gated sodium channel blockers such as mexiletine hydrochloride could be effective in reducing the severity of cold paresis. We designed the MEXiletine hydrochloride administration trial: a placebo-controlled, randomised, double-blind, multicentre, crossover study of the efficacy and safety in spinal and bulbar muscular atrophy (SBMA), also known as the MEXPRESS study. Although mexiletine hydrochloride administration has been shown to be effective in several neuromuscular diseases such as non-dystrophic myotonia and Machado-Joseph disease, its effectiveness as a symptomatic therapy for SBMA has not been confirmed to date. ${ }^{71617}$ This study is the first to evaluate the efficacy and safety of mexiletine hydrochloride administration on cold paresis in patients with SBMA.

\section{METHODS AND ANALYSIS}

\section{Ethical approval and trial registration}

This study will be conducted in compliance with the Helsinki Declaration and the Ethical Guidelines for Medical and Health Research Involving Human Subjects by the Japanese government and was approved. The study (protocol version 01.01.00) was registered with the University Hospital Medical Information Network (UMIN) Clinical Trials Registry (UMIN000026150) before the recruitment period began.

\section{Study design}

We followed the Standard Protocol Items for Randomised Trials recommendations when preparing the present protocol. ${ }^{18} 19$ This study is a multicentre, randomised, double-blind, placebo-controlled, $2 \times 2$ crossover, phase II trial to assess the safety and efficacy of mexiletine hydrochloride in patients with SBMA in accordance with the Consolidated Standards of Reporting Trials guidelines. ${ }^{20}$

\section{Recruitment and setting}

The study is being conducted in five Japanese centres located in universities, national centres and general hospitals. All participants will be recruited from the outpatient setting of these hospitals. Neurologists, who are investigators or coinvestigators, will identify patients with SBMA and will provide sufficient information about the explanatory statement. The neurologists will also provide the informed consent form to the candidates before they participate in the study. All participants will give their written informed consent for participation in the trial prior to screening. After the screening assessment for eligibility is completed, the neurologists will evaluate patient eligibility using a checklist and will register patients for this trial.

\section{Eligibility criteria}

After obtaining informed consent, potential participants will be screened for eligibility within 4 weeks prior to enrolment in this study by assessing vital signs, grip power, blood tests and ECG results. The inclusion and exclusion criteria are listed in box 1 .

\section{Intervention}

Participants will begin oral administration within 4 weeks after the screening. Each participant will take an overencapsulated $100 \mathrm{mg}$ of mexiletine hydrochloride (Boehringer Ingelheim $\mathrm{GmbH}$, Ingelheim, Germany) or lactose orally after every meal, three times a day for 4 weeks during intervention periods 1 and 2. Study participants will be assessed at least four times during the trial: on the start date of intervention period $1\left(\mathrm{~V}^{1}\right)$, on the completion date of intervention period $1\left(\mathrm{~V}^{2}\right)$, on the start date of intervention period $2\left(\mathrm{~V}^{3}\right)$ and on the completion date of intervention period $2\left(\mathrm{~V}^{4}\right)$. They will undergo neurological assessments, peripheral nerve conduction studies, motor function tests and pulmonary function tests and will complete several questionnaires. Drug treatment will be initiated after the baseline assessment $\left(\mathrm{V}^{0,2}\right)$. The participants will undergo efficacy assessments after 4 weeks in both periods $\left(\mathrm{V}^{1,3}\right)$. The participants will be asked about any adverse symptoms and their physical conditions using the interactive voice response (IVR) diary and via a telephone survey every week during both intervention periods $\left(\mathrm{T}^{1-6}\right)$. Mexiletine hydrochloride has a half-life of 10 hours in the blood. Therefore, we will establish a 7-day washout period between the two intervention periods (figure 1, table 1 ).

To improve adherence, participants will be asked to return any unused drug tablets and their daily medication diaries. To avoid the effect of other treatments when evaluating the effectiveness of this drug, participants will be prohibited from using the following medicines until the end of the evaluation period: luteinizing hormone-releasing hormone (LH-RH) agonists, LH-RH antagonists, testosterone drugs, 5-alpha-reductase inhibitors, antiandrogen drugs, protein anabolic hormones, progesterone drugs, oestrogen drugs, antiarrhythmic drugs, 


\section{Box 1 The inclusion and exclusion criteria}

\section{Inclusion criteria:}

1. Male patients with 38 or more cytosine, adenine and guanine (CAG) repeats in the androgen receptor gene.

2. Patients with muscle weakness due to lower motor neuron loss (bulbar palsy or extremity palsy).

3. Patients with paresis like sudden and relevant loss of force that affect activities of daily living of themselves when exposed to cold temperatures.

4. Patients between 20 and 80 years of age at the time of agreement to participate.

5. Patients who are capable of ambulatory hospital visits.

6. Patients whose hepatic and renal functions meet the following criteria: serum AST, ALT: $<4.0$ * upper limit of institutional reference value. Serum creatinine: $<1.5^{*}$ upper limit of institutional reference value.

7. Patients providing written informed consent.

\section{Exclusion criteria:}

1. Patients who have hypersensitivity to mexiletine hydrochloride.

2. Patients with second-degree atrioventricular block, third-degree atrioventricular block or left bundle branch block.

3. Patients with a Brugada-type ECG pattern.

4. Patients with a history of myocardial infarction, valvular disease or cardiomyopathy.

5. Patients whose heart rate is 50 /minute or less.

6. Patients with severe liver or renal dysfunction.

7. Patients with heart failure.

8. Patients whose systolic blood pressure is $90 \mathrm{~mm} \mathrm{Hg}$ or less.

9. Patients with ulnar neuropathy at the dominant hand.

10. Patients whose serum potassium level is $3.5 \mathrm{mEq} / \mathrm{L}$ or less.

11. Patients who take antiarrhythmic drugs.

12. Patients who take antiepileptic drugs.

13. Patient with neurological disorders other than spinal and bulbar muscular atrophy

14. Patients whose glycated haemoglobin National Glycohemoglobin Standardization Program (NGSP) level is $6.5 \%$ or more.

15. Patients who take hypoglycaemic drugs.

16. Patients with alcohol addiction.

17. Patients with drug addiction.

18. Patients who take cimetidine, rifampicin or theophylline.

19. Patients with severe complications such as serious infectious disease, postoperation, terminal cancer or debilitation.

20. Patients whose grip power in the dominant hand is less than $10 \mathrm{~kg}$.

21. Patients who are judged to be inappropriate to participate in the trial for any other reasons.

antiepileptic drugs, cimetidine, rifampicin, theophylline and any unapproved drugs. The examined drug will be discontinued if a patient is found to have a serum potassium level less than $3.5 \mathrm{mEq} / \mathrm{L}$ during the trial.

\section{Outcome measures}

The primary endpoint of this trial will be the longitudinal change in the difference in distal latencies of the ulnar nerve at the wrist in the dominant hand under room temperature and cold exposure conditions after 4 weeks in periods 1 and 2. Electrophysiological examinations of the peripheral nerves will be conducted using a Nicolet Viking EDX (Natus Medical Incorporated, Pleasanton,
California, USA). Peripheral nerve conduction studies of the ulnar nerve at the wrist will be performed at room temperature and after cold exposure. Cooling will be performed in the upper limbs for $7 \mathrm{~min}$ in cold water at $15^{\circ} \mathrm{C}^{21-25}$

Secondary outcome measures will include the quantitative muscle strength in the upper limbs (grip power, pinch power and a $10 \mathrm{~s}$ grip and release test ${ }^{26}$ ) both before and after cold exposure, tongue pressure, ${ }^{27}$ five components of the Quantitative Myasthenia Gravis (QMG) score (excluding the ptosis and diplopia sections) ${ }^{28}$ and a 15 -foot timed walk test. ${ }^{29}$ We will also evaluate the longitudinal change in the Revised Amyotrophic Lateral Sclerosis Functional Rating Scale $\mathrm{e}^{30-32}$ and the Spinal and Bulbar Muscular Atrophy Functional Rating Scale which is a validated, disease-specific scale with a high sensitivity to disease progression in SBMA. ${ }^{33}$ We will evaluate muscle mass with dual-energy X-ray absorptiometry (DXA) using fan-beam technology (Discovery A; Hologic, Bedford, Massachusetts, USA). The sum of the appendicular lean soft tissue mass measured with DXA has been validated by the skeletal muscle mass measurement using MRI and CT. ${ }^{34-36}$ We will also evaluate the 36-Item Short Form Health Survey, ${ }^{37}$ Individualised Neuromuscular Quality of Life questionnaire, ${ }^{38}$ IVR diary, ${ }^{16}{ }^{39}$ respiratory function values (vital capacity, forced vital capacity, percentage of forced expiratory volume in one second, peak expiratory flow, respiratory resistance and flow-volume curve ${ }^{40}$ and blood tests (complete blood count, electrolytes, creatinine, creatine kinase, ${ }^{41} 42$ aldolase, lactate dehydrogenase, aspartate aminotransferase, alanine aminotransferase and testosterone).

\section{Sample size calculation}

Under the assumptions of no carryover effect, no period effect and no interactions between patients, treatments and periods, we calculated the sample size based on a paired t-test. The expected difference in the primary endpoint between the two groups was set to 0.768 , and the $\mathrm{SD}$ was estimated to be 0.9 . Each pair of measurements in the same patient will have a correlation of approximately 0.35 . Based on these configurations, a sample size of 20 is required to reject the null hypothesis, such that the population means of the experimental and control groups are equal with a probability of 0.8 and a two-sided significance level of 0.05 .

\section{Randomisation and blinding}

Randomisation, stratified by the disease duration $(0-9 / \geq 10$ years from onset) and the CAG repeat size in the $A R$ gene (38-49/ $\geq 50$ number), will be performed with a computer-generated random code assigned by a central interactive web response system at Nagoya University Hospital. A double-blind study will be conducted to achieve a higher standard of scientific rigour in evaluating the efficacy and safety of the study agent. A medical doctor who specialises in clinical trials at the external facility will be delegated as the 'allocator', who is responsible for treatment 


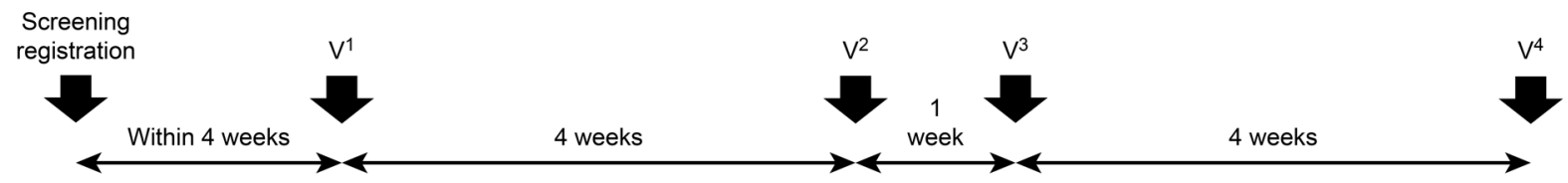

\begin{tabular}{|c|c|c|} 
Intervention period 1 & \multicolumn{2}{c}{ Intervention period 2 } \\
\hline Group A & Mexiletine hydrochloride 300 mg/day & Washout \\
\hline Group B
\end{tabular}

Figure 1 Flow diagram for the randomised controlled trial.

Table 1 Schedule of procedures, interventions and assessments

\begin{tabular}{|c|c|c|c|c|c|c|c|c|c|}
\hline & & & & $T^{1-3}$ & & 4-5Week & & $T^{4-6}$ & \\
\hline & Screening & Registration & $\mathbf{V}^{1}$ & (Telephone) & $\mathbf{v}^{2}$ & (Washout) & $\mathbf{V}^{3}$ & (Telephone) & $\mathbf{v}^{4}$ \\
\hline Agreement & ० & & & & & & & & \\
\hline Registration & & ० & & & & & & & \\
\hline Assignment & & 0 & & & & & & & \\
\hline Prescription & & & $\circ$ & & & & $\circ$ & & \\
\hline Collect leftover medicine & & & & & $\circ$ & & & & $\circ$ \\
\hline Daily medication diary & & & $\circ$ & & & & $\circ$ & & \\
\hline Grip power & ० & & ० & & $\circ$ & & o & & $\circ$ \\
\hline Pinch power & & & $\circ$ & & $\circ$ & & ○ & & $\circ$ \\
\hline 10 s grip and release test & & & $\circ$ & & $\circ$ & & ० & & $\circ$ \\
\hline Tongue pressure & & & $\circ$ & & $\circ$ & & ○ & & $\circ$ \\
\hline mQMG score & & & $\circ$ & & $\circ$ & & ० & & $\circ$ \\
\hline Timed walk test & & & $\circ$ & & $\circ$ & & ○ & & $\circ$ \\
\hline Peripheral nerve conduction studies & & & 0 & & ० & & ० & & $\circ$ \\
\hline Blood examination* & ० & & $\circ$ & & $\circ$ & & $\circ$ & & $\circ$ \\
\hline ECG & ० & & ० & & ० & & ० & & $\circ$ \\
\hline DXA & & o & & & & & & & \\
\hline Respiratory function test & & & 0 & & ० & & ० & & $\circ$ \\
\hline ALSFRS-R & & & $\circ$ & & ० & & $\circ$ & & $\circ$ \\
\hline SBMAFRS & & & $\circ$ & & $\circ$ & & ० & & $\circ$ \\
\hline SF-36 & & & $\circ$ & & $\circ$ & & ○ & & $\circ$ \\
\hline INQoL & & & ० & & ० & & ० & & $\circ$ \\
\hline IVR diary & & $\circ$ & ० & ○ & $\circ$ & & ० & $\circ$ & $\circ$ \\
\hline Vital signs & 0 & & 0 & & 0 & & 0 & & $\circ$ \\
\hline
\end{tabular}

${ }^{*}$ Complete blood count, electrolytes, creatinine, creatine kinase, aldolase, lactate dehydrogenase, aspartate aminotransferase, alanine aminotransferase, and testosterone.

ALSFRS-R, revised Amyotrophic Lateral Sclerosis Functional Rating Scale; DXA, dual-energy X-ray absorptiometry; INQoL, Individualised Neuromuscular Quality of Life questionnaire; IVR, interactive voice response; mQMG, Modified Quantitative Myasthenia Gravis; SBMAFRS, Spinal and Bulbar Muscular Atrophy Functional Rating Scale; SF-36, 36-Item Short Form Health Survey; $V^{1}$, intervention period 1; V

${ }^{2}$, intervention period $2 ; \mathrm{V}^{3}$, intervention period $3 ; \mathrm{V}^{4}$, intervention period 4. 
allocation. The allocator will ensure that it is impossible to determine whether the study agent is mexiletine hydrochloride or a placebo by its appearance or package. The allocator will ensure that the allocation code/list will be kept in a sealed envelope and that the allocation will be concealed before unblinding.

\section{Efficacy and safety/tolerability data analyses \\ Efficacy data analyses}

The primary endpoint of efficacy will be the longitudinal change in the difference in distal latencies of the ulnar nerve at the wrist in the dominant hand between the room temperature and cold exposure conditions at 4 weeks. In the primary analysis, we will examine the superiority of the mexiletine hydrochloride groups to the placebo-treated group using a mixed effect model for the primary endpoint. Secondary endpoints will be analysed using the mixed effect model, $\chi^{2}$ test, unpaired t-test and Wilcoxon signed-rank test depending on the characteristics of the data. All analyses will be conducted based on the intention-to-treat (ITT) principle, which will include all randomly assigned patients who received the study medication and provided at least one postbaseline efficacy datum, as well as the per-protocol set, which will include all ITT patients with no important protocol violations relevant to the assessment of the study agent efficacy. A two-sided value of $\mathrm{p}<0.05$ will be considered statistically significant. All statistical analyses will be performed using the SAS (V.9.4; SAS Institute, Cary, North Carolina, USA).

\section{Safety/tolerability data}

Safety will be evaluated in all patients who receive the study agent at least once. The safety and tolerability assessments including subjective and objective neurological symptoms including cold paresis, vital signs, medical examination findings, clinical laboratory data, ECG results and the intensity of adverse events (AEs) will be evaluated for each patient. Severe AEs will be defined as incapacitating or causing inability to work or undertake usual activities. Each AE will be coded to a preferred term and will be associated with an organ system according to an established and validated adverse reaction dictionary (MedDRA/J, V.18.0). An independent data and safety monitoring board will monitor AEs.

\section{Patient and public involvement}

We consulted patient representatives about the protocol development and recruitment. Prior to this trial, we performed peripheral nerve conduction studies preliminarily to determine the primary endpoint of this trial in subjects with SBMA with cold paresis who periodically attend the outpatient clinic. To enable subjects who participated in the preliminary study to join this trial preferentially, at the beginning, we will recruit them. We also informed and recruited participants through UMIN Clinical Trials Registry. For randomised controlled trials, the burden of the intervention will be assessed by patients themselves prior to participating in the trial.

\section{ETHICS AND DISSEMINATION}

\section{Research ethics approval}

Participants will provide signed informed consent before participating in the study. Participants will be able to withdraw from the study at any point. No reason for withdrawal will be required.

\section{Confidential}

Personal information about potential and enrolled participants will be stored in a manner that no third party may have access thereto.

\section{Dissemination}

The results of the study will be disseminated via several avenues including peer-reviewed journal publications, conference presentations, the UMIN Clinical Trials Registry and a summary of findings provided to the participants.

Ethics approval Ethics committee of Nagoya University Graduate School of Medicine, as a central institutional review board, and by each facility in Februaly and March in 2017.

\section{Author affiliations}

${ }^{1}$ Department of Neurology, Nagoya University Graduate School of Medicine, Nagoya, Japan

${ }^{2}$ Biostatistics Section, Centre for Advanced Medicine and Clinical Research, Nagoya University Graduate School of Medicine, Nagoya, Japan

${ }^{3}$ Department of Biostatistics and Bioinformatics, Graduate School of Medicine, The University of Tokyo, Tokyo, Japan

Acknowledgements We thank all patients with SBMA who participated and are concerned in this trial.

Contributors SY: drafting/revising the manuscript, research project execution, study design and concept. $\mathrm{AH}$ : revising the manuscript, research project execution and study design and concept. YH, TI and DI: research project execution. FK, MN, YK and $\mathrm{AH}$ : study design and concept. TN: research project execution and study design and concept. MK: research project organisation, revising the manuscript, research project execution and study design and concept.

Funding This work is supported by Nagoya University Hospital Funding for Clinical development.

Disclaimer The funder had no role in the study design; collection, management, analysis and interpretation of data; writing of the report; or the decision to submit the report for publication.

Competing interests None declared.

Patient consent Obtained.

Ethics approval Ethics committee of Nagoya University Graduate School of Medicine.

Provenance and peer review Not commissioned; externally peer reviewed.

Open access This is an open access article distributed in accordance with the Creative Commons Attribution Non Commercial (CC BY-NC 4.0) license, which permits others to distribute, remix, adapt, build upon this work non-commercially, and license their derivative works on different terms, provided the original work is properly cited, appropriate credit is given, any changes made indicated, and the use is non-commercial. See: http://creativecommons.org/licenses/by-nc/4.0/.

\section{REFERENCES}

1. Kennedy WR, Alter M, Sung JH. Progressive proximal spinal and bulbar muscular atrophy of late onset. A sex-linked recessive trait. Neurology 1968;18:671-80. 
2. Katsuno M, Tanaka F, Adachi H, et al. Pathogenesis and therapy of Spinal and Bulbar Muscular Atrophy (SBMA). Prog Neurobiol 2012;99:246-56.

3. Sorarù G, D'Ascenzo C, Polo A, et al. Spinal and bulbar muscular atrophy: skeletal muscle pathology in male patients and heterozygous females. J Neurol Sci 2008;264:100-5.

4. La Spada AR, Wilson EM, Lubahn DB, et al. Androgen receptor gene mutations in $\mathrm{X}$-linked spinal and bulbar muscular atrophy. Nature 1991;352:77-9.

5. Yu Z, Dadgar N, Albertelli M, et al. Androgen-dependent pathology demonstrates myopathic contribution to the Kennedy disease phenotype in a mouse knock-in model. J Clin Invest 2006;116:2663-72.

6. Araki K, Nakanishi H, Nakamura T, et al. Myotonia-like symptoms in a patient with spinal and bulbar muscular atrophy. Neuromuscul Disord 2015;25:913-5

7. Trivedi JR, Bundy B, Statland J, et al. Non-dystrophic myotonia: prospective study of objective and patient reported outcomes. Brain 2013;136:2189-200.

8. Straver DC, van Asseldonk JT, Notermans NC, et al. Cold paresis in multifocal motor neuropathy. J Neurol 2011;258:212-7.

9. Boulos PT, Heiman-Patterson TD, Alexander GM, et al. Patch clamp studies of the thr1313met mutant sodium channel causing paramyotonia congenita. Muscle Nerve 2000;23:1736-47.

10. Sugiura Y, Makita N, Li L, et al. Cold induces shifts of voltage dependence in mutant SCN4A, causing hypokalemic periodic paralysis. Neurology 2003;61:914-8.

11. Hodgkin AL, Katz B. The effect of temperature on the electrical activity of the giant axon of the squid. J Physiol 1949;109:240-9.

12. Dlouhá $H$, Donselaar $Y$, Teisinger J, et al. Effect of temperature and ouabain on th $\mathrm{Na}+--\mathrm{K}+$ activated membrane ATPase and electrogenic ionic pump of the golden hamster and mouse diaphragm. Physiol Bohemoslov 1980;29:543-52.

13. Rutkove SB. Effects of temperature on neuromuscular electrophysiology. Muscle Nerve 2001;24:867-82.

14. Webb J, Cannon SC. Cold-induced defects of sodium channel gating in atypical periodic paralysis plus myotonia. Neurology 2008;70:755-61.

15. Cannon SC. Channelopathies of skeletal muscle excitability. Compr Physiol 2015;5:761-90.

16. Statland JM, Bundy BN, Wang Y, et al. Mexiletine for symptoms and signs of myotonia in nondystrophic myotonia: a randomized controlled trial. JAMA 2012;308:1357-65

17. Kanai K, Kuwabara S, Arai K, et al. Muscle cramp in MachadoJoseph disease: altered motor axonal excitability properties and mexiletine treatment. Brain 2003;126:965-73.

18. Chan AW, Tetzlaff JM, Altman DG, et al. SPIRIT 2013 statement: defining standard protocol items for clinical trials. Ann Intern Med 2013;158:200-7.

19. Chan AW, Tetzlaff JM, Gøtzsche PC, et al. SPIRIT 2013 explanation and elaboration: guidance for protocols of clinical trials. BMJ 2013;346:e7586.

20. Schulz KF, Altman DG, Moher D, et al. CONSORT 2010 statement: updated guidelines for reporting parallel group randomized trials. Ann Intern Med 2010;152:726-32.

21. Streib EW, Sun SF, Yarkowsky T. Transient paresis in myotonic syndromes: a simplified electrophysiologic approach. Muscle Nerve 1982;5:719-23.

22. Streib EW. AAEE minimonograph \#27: differential diagnosis of myotonic syndromes. Muscle Nerve 1987;10:603-15.
23. Kuntzer T, Michel P. Muscle membrane polarisation after provocative tests, and after cooling: the normal CMAP changes to be expected. Clin Neurophysiol 2004;115:1457-63.

24. Fournier $\mathrm{E}$, Viala K, Gervais $\mathrm{H}$, et al. Cold extends electromyography distinction between ion channel mutations causing myotonia. Ann Neurol 2006;60:356-65.

25. Michel P, Sternberg D, Jeannet PY, et al. Comparative efficacy of repetitive nerve stimulation, exercise, and cold in differentiating myotonic disorders. Muscle Nerve 2007;36:643-50.

26. Ono K, Ebara S, Fuji T, et al. Myelopathy hand. New clinical signs of cervical cord damage. J Bone Joint Surg Br 1987;69:215-9.

27. Mano $\mathrm{T}$, Katsuno M, Banno $\mathrm{H}$, et al. Tongue pressure as a novel biomarker of spinal and bulbar muscular atrophy. Neurology 2014;82:255-62.

28. Barohn RJ, Mclntire D, Herbelin L, et al. Reliability testing of the quantitative myasthenia gravis score. Ann N Y Acad Sci 1998;841:769-72.

29. Brinkmann JR, Andres P, Mendoza M, et al. Guidelines for the use and performance of quantitative outcome measures in ALS clinical trials. J Neurol Sci 1997;147:97-111.

30. The amyotrophic lateral sclerosis functional rating scale. Assessment of activities of daily living in patients with amyotrophic lateral sclerosis. The ALS CNTF treatment study (ACTS) phase I-Il study group. Arch Neurol 1996;53:141-7.

31. Banno H, Katsuno M, Suzuki K, et al. Phase 2 trial of leuprorelin in patients with spinal and bulbar muscular atrophy. Ann Neurol 2009;65:140-50.

32. Katsuno M, Banno H, Suzuki K, et al. Efficacy and safety of leuprorelin in patients with spinal and bulbar muscular atrophy (JASMITT study): a multicentre, randomised, double-blind, placebocontrolled trial. Lancet Neurol 2010:9:875-84.

33. Hashizume A, Katsuno M, Suzuki K, et al. A functional scale for spinal and bulbar muscular atrophy: cross-sectional and longitudinal study. Neuromuscul Disord 2015;25:554-62.

34. Shih R, Wang Z, Heo M, et al. Lower limb skeletal muscle mass: development of dual-energy $\mathrm{X}$-ray absorptiometry prediction model. J Appl Physiol 2000;89:1380-6.

35. Bridge P, Pocock NA, Nguyen T, et al. Prediction of appendicular skeletal and fat mass in children: excellent concordance of dualenergy $\mathrm{X}$-ray absorptiometry and magnetic resonance imaging. $J$ Pediatr Endocrinol Metab 2009;22:795-804.

36. Zhao X, Wang Z, Zhang J, et al. Estimation of total body skeletal muscle mass in Chinese adults: prediction model by dual-energy X-ray absorptiometry. PLoS One 2013;8:e53561.

37. Fukuhara S, Bito S, Green J, et al. Translation, adaptation, and validation of the SF-36 health survey for use in Japan. J Clin Epidemiol 1998;51:1037-44.

38. Vincent KA, Carr AJ, Walburn J, et al. Construction and validation of a quality of life questionnaire for neuromuscular disease (INQoL). Neurology 2007;68:1051-7.

39. Statland JM, Wang Y, Richesson R, et al. An interactive voice response diary for patients with non-dystrophic myotonia. Muscle Nerve 2011;44:30-5.

40. Yamada S, Hashizume A, Hijikata Y, et al. Decreased peak expiratory flow associated with muscle fiber-type switching in spinal and bulbar muscular atrophy. PLoS One 2016;11:e0168846.

41. Hashizume A, Katsuno M, Banno H, et al. Longitudinal changes of outcome measures in spinal and bulbar muscular atrophy. Brain 2012;135:2838-48.

42. Hijikata Y, Katsuno M, Suzuki K, et al. Impaired muscle uptake of creatine in spinal and bulbar muscular atrophy. Ann Clin Trans/ Neurol 2016;3:537-46. 